\title{
Le dilemme de l'élevage ovin extensif dans les régions élevées du nord-ouest, semi-aride tunisien
}

\author{
M. Rekik ${ }^{1 *}$ M. Mahouachi ${ }^{1}$ M. Gharbi ${ }^{2}$ \\ W. Attia ${ }^{3}$ L. Medhioub ${ }^{1}$
}

Mots-clés

O vin - Céréale - Elevage extensif Production animale - Climat semiaride - Tunisie.

\begin{abstract}
Résumé
Deux sites de montagne à bioclimat semi-aride du nord-ouest de la Tunisie ont été choisis afin de caractériser les systèmes extensifs de production ovine. Les systèmes de production rencontrés ont été de type sédentaire, étroitement liés à la production céréalière ; I'usage des parcours a disparu au profit d'une extension de la culture d'orge accompagnée d'une utilisation de jachères sur des terres en pente de faible fertilité. De plus en plus confinés sur des espaces réduits, les troupeaux, en général de petite taille, étaient tributaires de stratégies alimentaires peu efficaces et étaient caractérisés par de faibles performances technico-économiques. Cet élevage était en outre handicapé par le soutien financier très précaire des agriculteurs-éleveurs, eux-mêmes vulnérables vis-à-vis des conditions climatiques et économiques. Face à des difficultés d'extension dans l'espace ou d'augmentation des effectifs, l'élevage ovin dans ces zones a été confronté à la nécessité d'accroître sa productivité. Des recommandations qui tiennent compte du contexte technico-économique particulier des exploitations agricoles de la zone sont proposées.
\end{abstract}

\section{INTRO DUCTION}

Les systèmes de production des petits ruminants dans l'étage bioclimatique semi-aride, fortement soumis aux effets des changements de l'environnement socio-économique et des restructurations foncières font face à des défis très importants comme le maintien du niveau de production, la rentabilisation des investissements publics en aménagements sylvo-pastoraux et la valorisation d'une incorporation de plus en plus grande de céréales secondaires et de sous-produits de meunerie dans la ration des animaux. Dans ces systèmes, les ovins sont une composante non dominante qui s'intègre aux systèmes de production agricole $(5,12)$. Ils assurent ainsi plus de stabilité au revenu des agriculteurs, ajoutent plus de souplesse quant au choix des options de gestion du système de production et représentent un signe extérieur du statut social au sein de la société rurale.

1. Laboratoire de production animale, Ecole supérieure d'agriculture du Kef, 7100 Le Kef, Tunisie

2. Laboratoire d'informatique et de statistiques, Ecole supérieure d'agriculture du Kef, 7100 Le Kef, Tunisie

3. Agence nationale pour la protection de l'environnement, 12 rue du Cameroun, 1002 Belvédère, Tunisie

* Auteur pour la correspondance

Tél. : 2168223086 ; fax : 2168223137 ; e-mail : rekik.mourad@iresa.agrinet.tn
Dans les régions semi-arides de montagne du nord-ouest de la Tunisie, les opportunités de revenus en dehors de l'agriculture sont très rares. La dégradation de la forêt et une croissance démographique soutenue ont entraîné une demande accrue en terres pour les cultures et l'élevage. Cette ressource de base dont la fragilité est grande, constitue une contrainte à l'intensification de l'agriculture. Ceci est d'autant plus vrai que la faible et variable réponse aux investissements rend leur utilisation financièrement risquée. Face à ce contexte, les agriculteurs-éleveurs de la zone de l'étude, qui œuvrent selon des stratégies très individualistes, se trouvent pris dans un cercle vicieux. S'ils persistent dans les modes de production actuels caractérisés par une tendance croissante à établir des cultures permanentes sur des terres marginales très fragiles et à accroître la pression animale, ils détruiront les bases d'une agriculture durable (à moyen et à long termes). Les formes extensives de production des ovins, autrefois à la base de l'agriculture de ces régions, se trouveraient ainsi menacées.

La présente étude, réalisée sur deux sites des régions d'altitude du nord-ouest semi-aride, a tenté d'aborder les systèmes de production des ovins par une description de l'environnement, une identification des contraintes et en proposant des mesures praticables dans le contexte particulier des exploitations ciblées. 


\section{ZONE DE L'ETUDE} ET METHODES UTILISEES

La présente étude à été réalisée dans le nord-ouest de la Tunisie dans les zones administratives du Kef et de Kasserine caractérisées par un bioclimat semi-aride (figure 1). Elle a concerné deux sites à haute altitude et distants d'environ $50 \mathrm{~km}$, localisés à la jonction des deux zones. Les deux sites, Ezzitouna dans la zone du Kef et Thala dans celle de Kasserine, se trouvent sur un grand carrefour de circuits de commercialisation des ovins reliant d'importants regroupements urbains au nord et au sud. Des débouchés plus lointains pour les ovins sont aussi recherchés, notamment à Tunis.

La zone de l'étude était historiquement un foyer de sédentarisation pour les anciennes communautés pastorales du nord (Jbelia) et du centre-ouest (Ayar). Aujourd'hui, elle est devenue un réservoir de départ vers le milieu urbain environnant. Dans la localité d'Ezzitouna, qui compte environ 4500 habitants, plus de 100 familles ont définitivement quitté la région pendant la dernière décennie et 200 ouvriers la quittent temporairement chaque année à la recherche d'un emploi.

La localité d'Ezzitouna ne disposant pas d'une station météorologique, les auteurs ont eu recours aux données enregistrées depuis une trentaine d'années dans la station la plus proche située au Ksour à $11 \mathrm{~km}$. La température moyenne annuelle dans cette localité est de $16,4{ }^{\circ} \mathrm{C}$ avec de très larges amplitudes thermiques

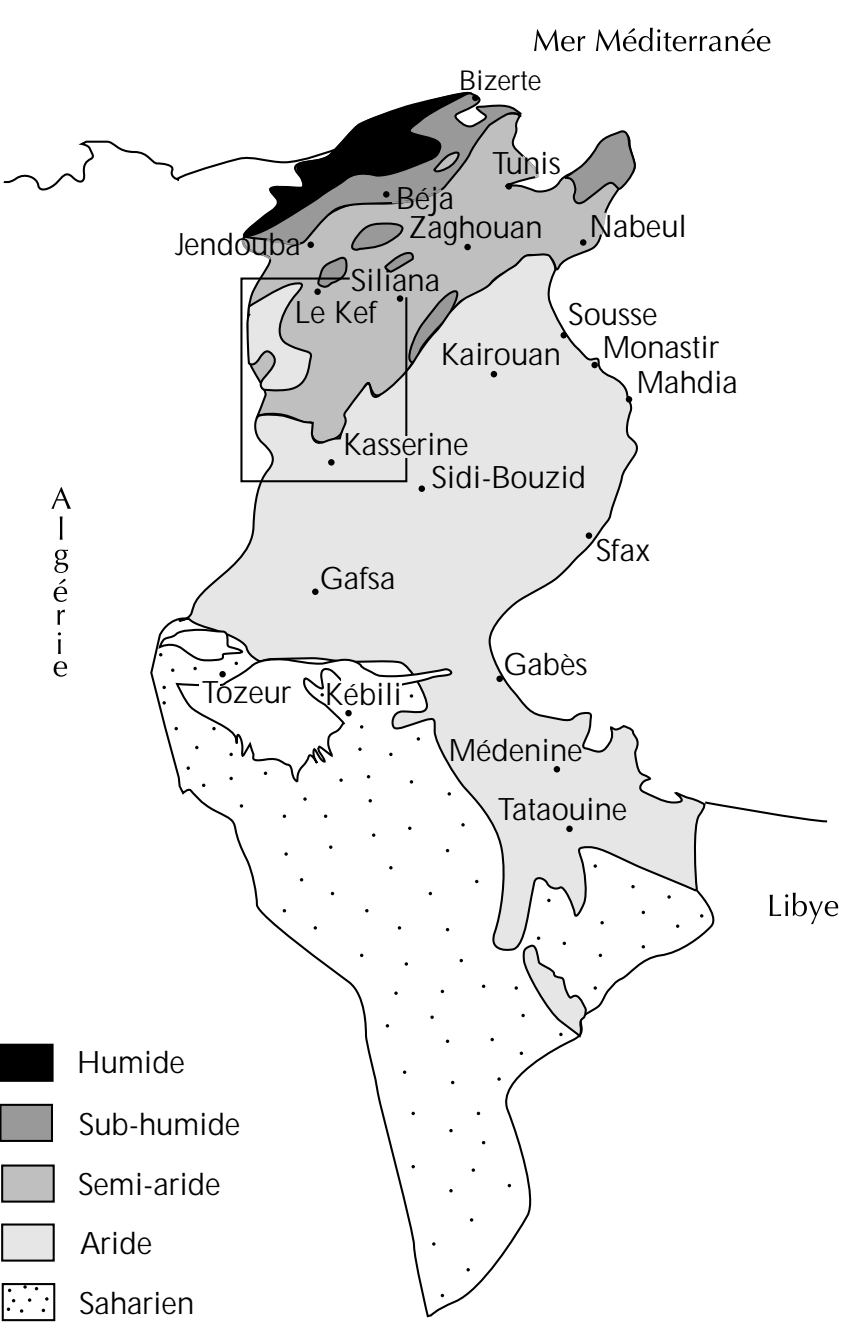

Figure 1 : carte des étages bioclimatiques en Tunisie avec en encadré une délimitation approximative de la zone de l'étude.
( $35^{\circ} \mathrm{C}$ en été et $-8^{\circ} \mathrm{C}$ en janvier). L'évaporation représente près de $1384 \mathrm{~mm}$ par an. Sur toute la zone de l'étude, la pluviométrie moyenne annuelle est de $400 \mathrm{~mm}$ avec des variations interannuelles de pluviosité considérables qui sont responsables de disettes périodiques et imprévisibles et qui constituent un obstacle à toute intensification soutenue des productions animales.

Le diagramme ombrothermique (figure 2) permet de situer la période de végétation active à l'aide d'une échelle où la quantité de pluie, exprimée en millimètres et divisée par deux, doit être supérieure à la température moyenne exprimée en degrés Celsius (1). Calculée de cette façon, cette période est de sept mois à Thala (de janvier à mai et de novembre à décembre). Dans la zone de l'étude, région élevée et continentale, les gelées matinales sont fréquentes du mois de novembre jusqu'en avril et doivent être prises en considération car ces nuits glaciales réduisent la croissance de l'herbe et entravent les cultures de primeurs et l'arboriculture.

Les différents types de sols relevés dans la zone de l'étude ont été :

- des sols minéraux bruts de type lithosol sur calcaire situés en altitude et des sols lithiques sur poudingue calcaire qui ne peuvent être mis en valeur que par reboisement et par plantation de réserves pastorales après un aménagement anti-érosif ;

- des sols calcimagnésiques rendziniformes et bruns calcaires à pente relativement douce et convenant aux cultures fourragères annuelles ;

- des sols peu évolués d'apport alluvial et colluvial dont l'extension est très limitée aux bas-fonds, aux abords des ravins et des cours d'eau. Ce sont les meilleurs sols céréaliers de la zone.

Les principaux groupements végétaux présents dans la zone sont situés à Artemesia et sont caractéristiques de l'étage bioclimatique semi-aride avec également l'apparition de faciès de dégradation dans les zones défrichées à Rosemarinus officinalis et Helianthemum cinereum et à Thymus algeriensis, Stipa retorta et Stipa paviflora (9).

Par ailleurs, la prospection des terres laissées en jachères, qui représentaient une ressource principale pour l'alimentation du cheptel, a révélé la dominance de Moricandia arvensis, Diplotaxis erucoides et Eruca vericaria. Ces espèces sont très peu ou pas du tout appétées par les ovins.

La caractérisation de l'environnement et l'étude des systèmes de production des ovins ont été réalisées à Ezzitouna et à Thala respectivement dans 47 et 102 exploitations, d'avril à juin 1997. Les éleveurs ovins ont été choisis au hasard parmi les petits (nombre de femelles inférieur à 20), les moyens (nombre de femelles compris entre 20 et 50 ) et les grands éleveurs (nombre de femelles supérieur à 50). Ces éleveurs ont répondu à un questionnaire reconstituant a posteriori les événements survenus pendant la cam-

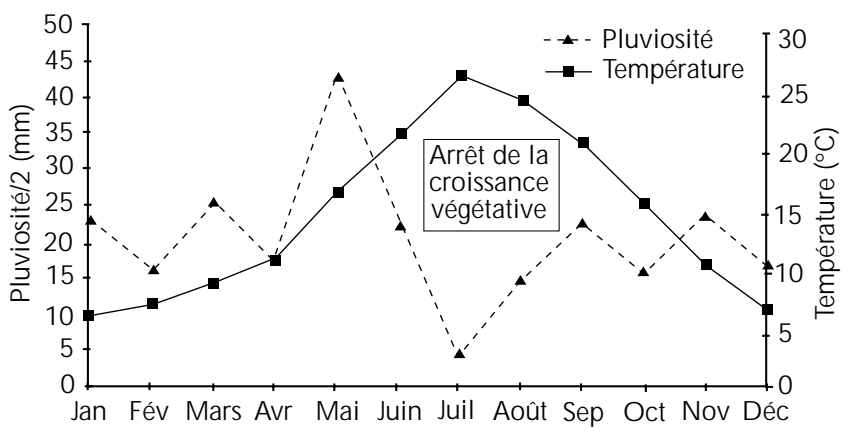

Figure 2 : diagramme ombrothermique permettant de situer (pendant dix ans, 1988-1998) les périodes de végétation active sur le site de Thala. 
pagne agricole de 1995-96 au cours de laquelle la pluviométrie a été moyenne, avec une bonne distribution mensuelle sur toute la zone de l'étude.

Afin de valider certaines informations révélées par les enquêtes sur les modes de production des ovins et les pratiques de gestion des ressources génétiques, dix élevages sur le site d'Ezzitouna ont été soumis à un suivi rapproché. Les élevages ont été choisis selon la prédisposition des éleveurs à soumettre leurs animaux aux différentes interventions et selon leur représentativité des trois classes de troupeaux désignées ci-dessus. La première année, les dix troupeaux comptaient 340 brebis ; ils sont passés à 364 brebis au début de la deuxième année de suivi. Le protocole de suivi, qui a duré 24 mois (années 1996 et 1997) et qui n'a concerné que le troupeau de brebis et les jeunes nés pendant le suivi, a comporté un contrôle de la croissance des agneaux et des naissances tous les 21 jours de novembre à mai et des relevés mensuels de la dynamique des troupeaux. Les événements en rapport avec la reproduction des troupeaux (agnelage, taille des portées) ou les mortalités (jeunes et adultes) ont été notés mensuellement pendant les deux années de suivi.

\section{Paramètres suivis}

L'environnement de production et les systèmes d'élevage des ovins pratiqués ont été définis selon :

- les caractéristiques des exploitations et l'occupation des sols ;

- la structure des troupeaux ovins et les modes d'élevage ;

- la place de l'orge dans l'alimentation des troupeaux ;

- les paramètres zootechniques de cet élevage ;

- les modes de production de la viande ovine.

\section{Traitement et édition des données}

Après l'édition des données de base, 26 enquêtes sur le site de Thala et huit sur celui d'Ezzitouna ont été écartées. Elles ont concerné pour la plupart des exploitants nouvellement attributaires de lots d'ovins dans le cadre des programmes régionaux de développement rural qui avaient, par conséquent, peu de recul en matière d'élevage ovin et qui n'appartenaient donc pas à la classe d'éleveurs ciblés par cette étude. Dans les enquêtes restantes ont été écartées celles dont le nombre d'ovins a été très réduit $(<10)$ ou celles pour lesquelles les informations ont été jugées insuffisantes.
Par ailleurs, la mise en œuvre du contrôle de croissance a été, dans certains cas, perturbée par la vente très précoce des agneaux parfois accompagnés de leurs mères. Ces ventes, bien qu'elles aient pu priver cette étude d'une information plus volumineuse relative aux paramètres de croissance des agneaux, ont été révélatrices de certaines pratiques sur les modes d'écoulement des produits. Les paramètres de croissance ont été calculés en utilisant le logiciel Ovins des services du contrôle des performances (Office de l'élevage et des pâturages, ministère de l'Agriculture).

\section{RESU LTATS}

\section{Caractéristiques des exploitations et occupation des sols}

Les données sur l'occupation des sols (tableau I) ont montré que l'agriculture dans la région de l'étude était principalement pluviale puisque les superficies irriguées ont représenté moins de 5 p. 100 de la superficie totale pour une superficie moyenne des exploitations respectivement de 45,7 et de 28 ha à Ezzitouna et à Thala. Sur les deux sites, le système de production a été dominé par un assolement biennal : céréales (52-57 p. 100 de la superficie totale) et jachères (27-29 p. 100 de la superficie totale). L'orge a été la culture la plus importante occupant 35 et 33 p. 100 de la superficie totale respectivement à Ezzitouna et à Thala.

Le faible recours aux cultures fourragères $(2,4$ et 3,8 p. 100 de la superficie totale respectivement à Ezzitouna et à Thala) et le peu d'espace laissé aux parcours (seulement 2,5 p. 100 de la superficie totale à Thala) ont été d'autres caractéristiques importantes de l'occupation du sol étant donné leur rapport direct avec le système alimentaire des troupeaux.

\section{Structure des troupeaux ovins et modes d'élevage}

Sur les deux sites d'Ezzitouna et de Thala, le système d'élevage a été sédentaire, très extensif et orienté vers la production de viande. Les éleveurs ont été généralement des personnes âgées pratiquant l'agriculture et l'élevage. Seulement 10 p. 100 des chefs d'exploitation ont été âgés de 33 à 45 ans alors que la majorité (54 p. 100) avait 60 à 90 ans. Sur les 115 éleveurs enquêtés, 41 (35 p. 100) seulement ont eu recours à une main d'œuvre rémunérée qui est intervenue dans les tâches quotidiennes de conduite des troupeaux. Cette pratique a été liée à la taille du troupeau ; elle a été plus répandue dans les élevages dont l'effectif a été supérieur à 50 femelles.

\section{Tableau I}

O ccupation des sols et caractéristiques moyennes des exploitations pendant la campagne agricole de 1995-96 sur les sites d'Ezzitouna $(n=39)$ et de Thala $(n=76)$

\begin{tabular}{lcccc} 
& \multicolumn{2}{c}{ Ezzitouna } & & Thala \\
& Superficie (ha) & \% de superficie totale & Superficie (ha) & \% de superficie totale \\
\hline Superficie totale & 1785 & 100 & 2134 & 100 \\
en sec & 1749 & 97,99 & 2022 & 94,7 \\
en irriguée & 36 & 2,01 & 112 & 5,3 \\
Occupation des sols & & & 527 & 24,70 \\
Blé & 304 & 17,03 & 711 & 33,31 \\
Orge & 627 & 35,12 & 115 & 5,38 \\
Arboriculture & 98 & 5,49 & 632 & 29,61 \\
Jachère & 483 & 27,05 & 15 & 0,7 \\
Légumineuses & 22 & 1,23 & 81 & 3,80 \\
Cultures fourragères & 43 & 2,40 & 53 & 2,50
\end{tabular}


Le système de conduite pratiqué $a$, en fait, nécessité peu d'interventions sauf au moment de la tonte. Pour la prévention sanitaire $\mathrm{du}$ troupeau, les éleveurs ont eu principalement recours aux campagnes étatiques gratuites de lutte contre les maladies infectieuses comme la fièvre aphteuse, la clavelée et la brucellose. Pour les autres traitements, 59 p. 100 des éleveurs sur les deux sites ont consacré des montants annuels variables aux soins vétérinaires, avec notamment des dépenses par brebis par an positivement corrélées $(\mathrm{r}=0,63, \mathrm{P}<0,05)$ au nombre de femelles sur l'exploitation.

La composition du cheptel sur les deux sites a été dominée par les ovins (tableau II). Les caprins ont été peu nombreux et la présence des bovins, plutôt récente, a été surtout limitée aux exploitations où il y avait de l'irrigation. L'élevage ovin à viande dans les zones montagneuses du nord-ouest semi-aride a dominé toutes les autres formes d'élevage.

Chaque élevage a été composé en moyenne respectivement de 40 et de 33 femelles ovines pour des superficies d'exploitation de 45,7 et de 28 ha à Ezzitouna et à Thala. Sur les deux sites, la majorité des élevages ont été de petite et de moyenne taille (effectifs en dessous de 50 femelles ovines) (tableau III). Le génotype ovin dominant dans la zone de l'étude a été le mouton à queue fine de l'Ouest, race prépondérante dans la région des hauts plateaux du nord-ouest tunisien. Les animaux de cette race sont hauts sur pattes, adaptés au pâturage sur pentes et ils ont une toison presque blanche d'une qualité moyenne. Sur le site de Thala, les animaux ont tous été de la race à queue fine de l'Ouest. En revanche, sur celui d'Ezzitouna, une plus grande variabilité a été notée faisant apparaitre trois classes. Plus de la moitié des animaux (57 p. 100) ont été conformes au modèle standard de la race à queue fine de l'Ouest défini dans la littérature (15), 21 p. 100 ont été du type Barbarine, animal à grosse queue qui caractérise l'élevage ovin sur steppes en Tunisie et le reste (22 p. 100) a été composé de croisements très divers avec un appendice caudal intermédiaire entre la queue fine et la Barbarine et avec recherche d'une pigmentation noire.

\section{Place de l'orge dans l'alimentation des troupeaux}

La faible productivité des céréales liée à une pluviosité insuffisante et irrégulière a constitué la principale contrainte du système étudié. Plus cette contrainte a été grande, moins la production végétale dans le système a été importante et plus la place de l'élevage dans la part des revenus et comme moyen de subsistance a été prépondérante. Il a par ailleurs été difficile, pour de courtes périodes d'observation, d'apporter des informations précises sur les aspects liés au bilan fourrager ou à la charge animale par unité de surface.

L'alimentation du cheptel, outre les achats à l'extérieur, est surtout provenue des terres laissées en jachères ou emblavées en fourrages. Elle a peu varié, l'orge ayant été presque le seul fourrage adopté. Il a été utilisé pour le pâturage au vert, pour la production de foin lorsque la biomasse a été suffisante et pour la production de grains destinés à l'engraissement des agneaux, comme complément saisonnier ou comme complément de sauvegarde.

Ainsi, sur le site d'Ezzitouna, des pratiques similaires dans le mode de valorisation des terres ont permis d'élaborer une formule empirique pour l'estimation de la superficie réservée à l'alimentation des animaux (Sraa). Celle-ci a été calculée à partir de la superficie en propriété réservée aux cultures d'assolement (Spca), les parcours $(\mathrm{Sp})$, les terres en location $(\mathrm{Sl})$ et la terre en association (Sa). La formule élaborée s'écrit : $\mathrm{Sraa}=\mathrm{Sp}+\mathrm{Sl}+1 / 3 \mathrm{Spca}+1 / 3$ $(2 / 3 \mathrm{Spca}+1 / 2 \mathrm{Sa})$, chaque éleveur ayant en général consacré un tiers de sa propre terre à la jachère, un tiers à la terre emblavée en orge et un tiers aux cultures d'assolement. En outre, les éleveurs se sont fréquemment associés pour 50 p. 100 des terres et ont consacré toutes les emblavures d'orge à l'alimentation du bétail.

\section{Tableau II}

Composition des élevages pendant la campagne agricole de 1995-96 dans les exploitations enquêtées sur les sites d'Ezzitouna $(n=39)$ et de Thala $(n=76)$

\begin{tabular}{lccrc} 
& \multicolumn{2}{c}{ Ezzitouna } & \multicolumn{2}{c}{ Thala } \\
\cline { 2 - 5 } & $\begin{array}{l}\text { Nb. total de } \\
\text { Femelles }\end{array}$ & $\begin{array}{c}\text { Nb. moyen de } \\
\text { femelles par } \\
\text { exploitation }\end{array}$ & $\begin{array}{c}\text { Nb. total de } \\
\text { femelles }\end{array}$ & $\begin{array}{c}\text { Nb. moyen de } \\
\text { femelles par } \\
\text { exploitation }\end{array}$ \\
O vins & 1481 & 40,2 & 2495 & 32,8 \\
Caprins & 86 & 2,3 & 250 & 3,3 \\
Bovins & 92 & 2,4 & 51 & 0,7
\end{tabular}

\section{Tableau III}

Distribution des élevages ovins enquêtés sur les sites d'Ezzitouna et de Thala en fonction du nombre de femelles ovines (campagne agricole de 1995-96)

Classe d'élevage (nb. de femelles ovines)

\section{Ezzitouna}

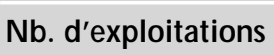

$<20$

20-50

$>50$

Total

16

15

8

39
$\%$

41,0

38,5

20,5

100
Thala

N b. d'exploitations $\%$ 


\section{Paramètres zootechniques de l'élevage ovin}

Le tableau IV résume quelques-uns des paramètres zootechniques des troupeaux ovins sur les deux sites. Sur le site d'Ezzitouna, les paramètres ont été établis dans le cadre du suivi des performances des troupeaux, alors que sur celui de Thala, ils ont été estimés à partir des enquêtes et doivent, par conséquent, être manipulés avec précaution.

Dans la zone de l'étude, la pratique de garder en permanence les mâles dans le troupeau a conduit à un échelonnement des naissances tout au long de l'année (figure 3) avec un pic en automne (40 p. 100 des agneaux nés entre septembre et novembre) et un autre de moindre importance au printemps (20 p. 100 des agneaux nés en avril et en mai).

La fertilité des troupeaux ovins sur les deux sites a été en moyenne respectivement de 85,4 et de 91 p. 100 sur les sites d'Ezzitouna et de Thala (tableau IV) tout en signalant que pour le premier site la valeur rapportée a représenté la moyenne de deux années consécutives. La mortalité des jeunes de la naissance jusqu'au sevrage n'a pas dépassé les normes généralement admises pour les races rustiques allaitantes avec des valeurs respectivement de 5,7 et 4 p. 100 sur les sites d'Ezzitouna et de Thala. Il en a été de même pour la mortalité des adultes dont la valeur moyenne pour les deux années de suivi à Ezzitouna n'a pas dépassé 2 p. 100. Pour la prolificité, des valeurs très proches ont été rapportées pour les deux sites (tableau IV). Les niveaux modérés de prolificité enregistrés ont aussi été conformes aux valeurs couramment rapportées dans les élevages contrôlés de la base de sélection et ont confirmé les potentialités limitées des races locales Barbarines et à queue fine de l'Ouest pour ce paramètre.

Pour ce qui concerne la gestion des ressources génétiques des troupeaux, de faibles taux de réforme des brebis à Ezzitouna $(10,2$ p. 100) et à Thala $(5,9$ p. 100) ont été rapportés. Les normes pour ce paramètre se situent entre 20 et 25 p. 100 pour conserver un bon équilibre de la pyramide des âges du troupeau. L'aspect de cette dernière sur le site d'Ezzitouna (figure 4) a mis en évidence l'effet de la sécheresse sur le renouvellement du troupeau. En effet, les effectifs réduits de femelles âgées de 2 et 3 ans dans les élevages correspondent à des renouvellements pendant les années 1993 à 1995, période très sèche dans la région. Si l'on prend en compte la mortalité des adultes, la réforme d'animaux et l'introduction de nouveaux sujets dans les troupeaux, le taux d'accroissement numérique moyen dans les dix élevages contrôlés du site d'Ezzitouna a atteint 7,05 p. 100.

Dans un système de production de viande, la croissance des agneaux, en plus des résultats de reproduction des troupeaux, est un facteur déterminant de la rentabilité des élevages. Les niveaux de croissance des agneaux ont été calculés pendant deux années consécutives sur le site d'Ezzitouna avec un effectif total de 452 individus. Les croissances sont présentées au tableau IV séparément pour les mâles et les femelles par tranches d'âge entre 10

\section{Tableau IV}

Paramètres zootechniques des troupeaux ovins sur les sites d'Ezzitouna et de Thala

$\begin{array}{lcc}\text { Paramètre zootechniques } & \text { Ezzitouna * } & \text { Thala ** } \\ \text { Taux de fertilité (\%) } & 85,4 & 91 \\ \text { Taux de prolificité (\%) } & 113 & 111 \\ \text { Taux de mortalité des jeunes (\%) } & 5,7 & 4 \\ \text { Taux de mortalité des adultes (\%) } & 1,94 & - \\ \text { Taux de réforme (\%) } & 10,2 & 5,9 \\ \text { Croissance entre } 10 \text { et } 30 \text { jours d'âge des agneaux (g/jour) } & 165 \pm 39,5 & - \\ \text { Croissance entre } 30 \text { et } 70 \text { jours d'âge des agneaux (g/jour) } & 121 \pm 60,1 & - \\ \text { Croissance entre } 10 \text { et } 30 \text { jours d'âge des agnelles (g/jour) } & 145 \pm 63,6 & - \\ \text { Croissance entre } 30 \text { et } 70 \text { jours d'âge des agnelles (g/jour) } & 106 \pm 50,2 & - \\ \text { Poids à } 70 \text { jours des agneaux (kg) } & 15,5 \pm 3,49 & - \\ \text { Poids à } 70 \text { jours des agnelles (kg) } & 14,3 \pm 3,40 & -\end{array}$

* Valeurs calculées dans dix élevages au terme d'un contrôle des performances pendant 24 mois (1996 et 1997)

** Valeurs estimées de la campagne agricole de 1995-96 à travers les enquêtes (au dire d'acteur)

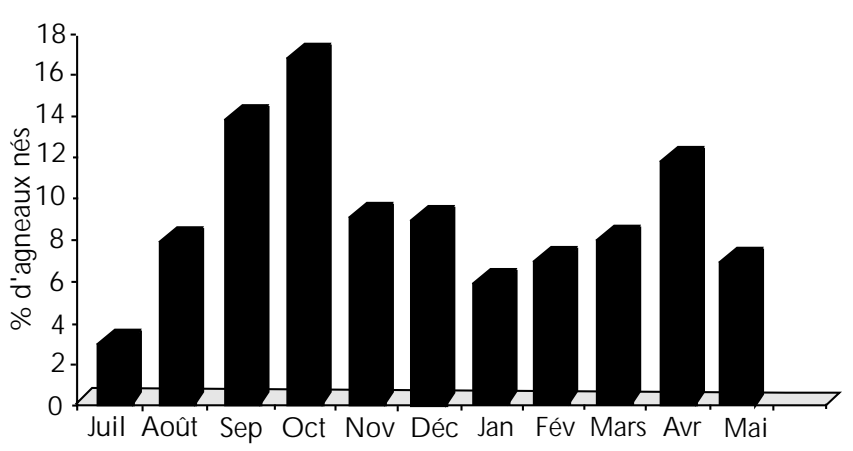

Figure 3 : distribution des naissances en 1996 et 1997 dans les troupeaux ovins sur le site d'Ezzitouna (nord-ouest, semiaride tunisien).

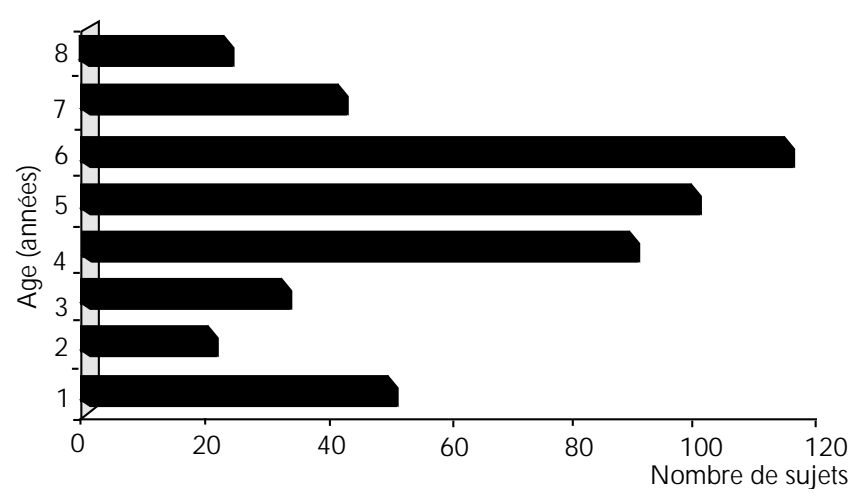

Figure 4 : pyramide des âges des brebis en 1996 et 1997 dans 39 troupeaux ovins sur le site d'Ezzitouna (nord-ouest, semi-aride tunisien). 
et 30 jours et entre 30 et 70 jours. A 70 jours d'âge, les agneaux mâles et femelles ont respectivement pesé $15,5 \pm 3,49$ et 14,3 $\pm 3,40 \mathrm{~kg}$. Toutefois, des différences importantes ont été observées entre les deux années avec des poids moins élevés la première année, respectivement $12,4 \pm 2,16$ et 12,3 $\pm 2,41 \mathrm{~kg}$ pour les mâles et les femelles. Les valeurs correspondantes ont atteint 17,2 $\pm 2,97$ et $15,1 \pm 3,50 \mathrm{~kg}$ la deuxième année. Les valeurs plus élevées de la croissance pendant la première tranche d'âge pour les deux sexes, fortement corrélées à la production laitière des mères, sont caractéristiques des races ovines allaitantes qui évoluent dans les milieux difficiles des zones arides et semi-arides.

\section{Modes de production de la viande ovine}

Pour les deux sites de l'étude, les lieux de production de l'agneau ont été très ouverts sur un vaste réseau de circuits de commercialisation le plus souvent courts. Le principal produit a été l'agneau léger vendu à un âge de 3 à 5 mois avant même d'être sevré et dont le poids vif a souvent été compris entre 18 et $25 \mathrm{~kg}$. Sur le site de Thala, 47 p. 100 environ des agneaux produits ont été écoulés avant sevrage. Cette tendance a été confirmée par la dynamique d'écoulement des agneaux nés entre octobre et décembre sur le site d'Ezzitouna et par la présence des sujets à l'engraissement sur les exploitations pendant la même période (figure 5). La grande importance des ventes entre avril et mai a été liée, d'une part, à la célébration de la fête musulmane du sacrifice et, d'autre part, au choix des éleveurs d'alléger la charge sur l'exploitation à l'approche de la saison estivale annonciatrice d'une diminution brusque des ressources alimentaires naturelles.

Sur les sites de Thala et d'Ezzitouna, l'engraissement des agneaux a représenté une forme de diversification de l'élevage ovin. Il s'agit plutôt, dans ce type de système, d'une prise de poids que d'engraissement au sens propre du terme. Il y avait des agneaux produits dans le troupeau mais également des sujets maigres achetés sur les marchés et alourdis pour viser des périodes fastes d'écoulement (le mois Saint, la fête de sacrifice) sur les marchés de Tunis et autour des grandes zones urbaines. Dans cette étude, aucun suivi technique des ateliers d'engraissement n'a été effectué. En revanche, l'importance de ce type de production et la place qu'il occupe dans la production de viande ont fait partie des objectifs de l'étude. Parmi les éleveurs enquêtés, 28 et 34 p. 100 respectivement à Thala et à Ezzitouna ont eu des ateliers d'engraissement. En moyenne, la taille de ces ateliers ont été de 31 et 24 têtes respectivement à Thala et à Ezzitouna avec un minimum de 8 et un maximum de 110 têtes. Ces ateliers ont généralement été constitués à la fin du printemps ou au début de l'été, période au cours de laquelle les sujets ont continué de pâturer sur les chaumes. L'intro-

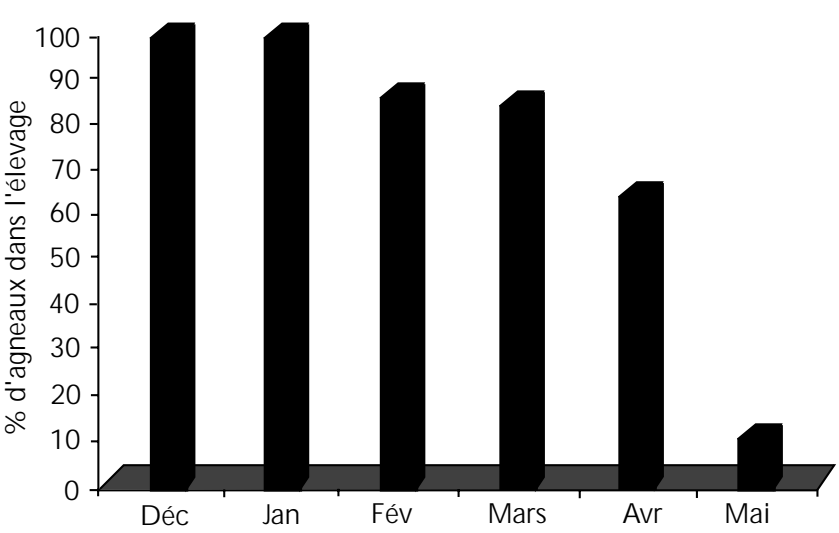

Figure 5 : dynamique d'écoulement des agneaux en 1996 et 1997 dans 39 troupeaux ovins sur le site d'Ezzitouna (nordouest, semi-aride tunisien). duction de céréales secondaires (l'orge essentiellement) a été progressive et a pu atteindre des quantités supérieures à $1,2 \mathrm{~kg}$ par tête et par jour à la fin de la phase d'engraissement (les deux ou trois derniers mois) qui s'est entièrement déroulée en bergerie. Des quantités très réduites de fourrages ont été distribuées (entre 200 et $400 \mathrm{~g}$ de foin ou de paille par tête et par jour) et aucune autre ressource alimentaire comme les suppléments azotés ou les suppléments à base de minéraux et de vitamines n'a été incorporée.

En plus des initiatives individuelles, certaines formes associatives pour la mise en place des ateliers d'engraissement ont été rapportées sur les deux sites, comme celle où des émigrants originaires des sites financent l'achat des agneaux maigres.

\section{DISCUSSION}

En référence à la classification des systèmes d'élevage établie par la Fao (13), la production des ovins dans la zone ciblée par cette étude s'apparente au large système pluvial des zones arides et semi-arides avec, comme le rapporte Flamant (7) pour le sud du Bassin méditerranéen, une extension de la culture des céréales et un accroissement de la charge au pâturage menaçant la pérennité des ressources naturelles et l'équilibre des milieux. En Tunisie, il est certain que les parcours pendant ces dernières décennies ont de moins en moins contribué à l'alimentation du cheptel des filières extensives et que leur extension spatiale est en nette diminution (14), conséquence de l'apurement foncier et de l'expansion des cultures. Il faudrait, néanmoins, dans le cas particulier de cette étude, tenir compte lors de l'interprétation de ces chiffres, de l'ambiguité dans la désignation des zones de parcours, comme la confusion entre les terres de jachères et les terres marginales à faible rendement céréalier. Une plus grande rigueur méthodologique n'a pas été possible pendant les enquêtes pour cerner avec exactitude la superficie des terres de parcours. C'est ainsi que les auteurs ont pu observer, notamment sur le site d'Ezzitouna où les parcours ont encore représenté 12 p. 100 de la superficie agricole totale, que ce qui était désigné comme parcours était généralement formé par les terres les plus marginales sur pentes accentuées, dans les ravins ou à proximité des cours d'eau. De plus, les éleveurs ont semblé peu soucieux de l'état des parcours, de leur préservation et de leur amélioration.

Il est par ailleurs important de noter que les améliorations des parcours dans la zone de l'étude, basées sur une approche non participative, ont échoué. Il ne restait que très peu de traces d'arbustes fourragers ou de cactus inermes plantés. Plusieurs des bénéficiaires des programmes d'amélioration pastorale rencontrés ont reproché aux structures techniques, d'une part, l'inadaptation des espèces plantées aux conditions climatiques de la région (froid, gelées et sécheresse) et, d'autre part, l'absence de suivi technique. Il semble que la disparition des plantations et des aménagements pastoraux a ainsi contribué à dissocier davantage les systèmes d'élevage ovin de l'utilisation des parcours.

L'accroissement de la demande en orge pour l'alimentation animale expliquerait, en grande partie, comment les éleveurs ont pu faire face aux aléas climatiques et à la réduction des parcours. Ainsi, selon la Banque mondiale (14), le disponible annuel en orge-grain pour le bétail en Tunisie a triplé entre 1963-65 et 199193 en raison d'une progression remarquable de la production. Les facilités de culture, de récolte, de stockage et d'utilisation par rapport aux autres fourrages de complément (foin, ensilage, cactus, arbustes fourragers) justifient l'extension de l'orge comme aliment de bétail notamment dans les petites et moyennes exploitations. L'association orge-élevage ovin est forte, puisque les corrélations 
de la surface de l'orge avec les unités ovines sont nettement supérieures à celles des unités bovines des régions semi-arides du Maroc (4).

L'extension de la culture d'orge, si elle a permis de sécuriser les besoins alimentaires des animaux à l'échelle de la zone de l'étude, risque de porter un préjudice irréparable au milieu lorsqu'elle s'étend aux sols fragiles des régions semi-arides. Désormais, ce processus semble irréversible et seules des suggestions peuvent être émises pour tenter d'en améliorer l'efficience en minimisant l'impact sur le milieu physique. C'est ainsi que les auteurs préconisent :

- d'orienter, autant que possible, les éleveurs vers des cultivars d'orge plus appropriés à l'exploitation par les animaux (appétibilité, tolérance vis-à-vis de la sécheresse, richesse en matière azotée, résistance au piétinement, adaptation à la double exploitation, etc.) (11) ;

- de préserver les sols fragilisés par des techniques douces de conservation des eaux et du sol comme les bandes en herbes et de pratiquer la culture d'orge dans l'interbande (alley cropping) ;

- d'utiliser les engrais avec la culture d'orge. Dans les régions semi-arides de Syrie, il a été montré que la fertilisation au niveau du lit de semences stimule un développement précoce de la plante et améliore l'efficacité d'utilisation de l'eau (2). Les auteurs estiment qu'à long terme cette pratique est rentable avec un niveau acceptable de risque.

Ce système de production céréalier basé sur l'orge est valorisé par des troupeaux ovins de petite taille avec très souvent des effectifs en dessous de 50 unités femelles. La tendance actuelle dans la zone de l'étude est vers l'émergence des petits élevages. Ceci rejoint les conclusions de l'étude de la Banque mondiale (14) et les résultats de l'enquête nationale sur les structures des exploitations (6). Il a été retenu qu'à l'échelle nationale les systèmes d'élevage ovin évoluent vers une plus forte concentration d'animaux dans les petites exploitations détenues par une majorité de petits éleveurs (environ 75 p. 100) dont la taille des élevages varie de 1 à 20 unités femelles. Cette émergence de petits élevages est la résultante de deux phénomènes, l'accroissement du nombre de nouveaux petits éleveurs et le transfert d'effectifs des grandes vers les petites et moyennes exploitations, conséquence de l'atomisation progressive des anciennes communautés pastorales et du partage des terres collectives. Avec ces petits élevages, les efforts des services de la vulgarisation deviennent de plus en plus difficiles et plusieurs des éleveurs qui ont participé au questionnaire ont reproché au système de vulgarisation actuel de recommander des références techniques financièrement non réalisables.

Le mode d'élevage de ces petits troupeaux est marqué par un caractère très extensif et par un faible investissement de la part des agriculteurs-éleveurs. La précarité des logements pour les animaux, la quasi-inexistence d'équipements d'élevage ou le nonrecours à l'achat de béliers sélectionnés sont d'autres indices du caractère extensif du système de production. Les actuels agriculteurs-éleveurs dans la zone de l'étude investissent moins d'énergie et de moyens dans l'élevage ovin pour adopter des stratégies très diverses concernant en particulier les céréales, l'arboriculture, le maraîchage, les bovins laitiers, ou des stratégies extra-agricoles comme le commerce, le transport, le travail journalier. A terme, cette individualisation a engendré la perte de connaissances précieuses en matière de gestion de l'environnement et les ressources alimentaires et les troupeaux sont moins bien entretenus.

La médiocrité des performances de production (gains moyens quotidiens des agneaux), liée à ce caractère extensif du mode de conduite des troupeaux, n'est cependant pas vérifiée au niveau des paramètres de reproduction et de mortalité des jeunes qui sont d'un bon niveau. Il faut toutefois souligner que des différences importantes ont été notées entre les élevages de la zone de l'étude pour les paramètres reproductifs rapportés. Dans plusieurs cas, des valeurs faibles ont été constatées dont l'amélioration peut être obtenue par des mesures simples et peu coûteuses comme l'adéquation des apports alimentaires aux besoins des animaux pendant les phases critiques du cycle de production. Cette même recommandation est également valable pour l'amélioration des performances de croissance des agneaux dont les grandes différences résultent d'importantes variations inter- et intra-élevages mais sont également liées à l'effet prépondérant de l'année. L'inadéquation entre les apports alimentaires et les besoins physiologiques des animaux est la conséquence du mode de conduite de la reproduction qui est gérée indépendamment des conditions du milieu pour ce qui concerne notamment le disponible alimentaire. Dans des milieux difficiles similaires, les éleveurs peuvent sauter un cycle reproductif pour limiter les pertes de reproductrices épuisées par la lactation en l'absence de nourriture suffisante (3). L'échelonnement des mises bas tout au long de l'année, même s'il peut léser certains individus dans le troupeau, pourrait représenter un choix délibéré de l'éleveur visant un usage plus souple de la main d'œuvre familiale et l'utilisation pendant toute l'année des produits comme fonds de sécurité. Il constituerait une assurance antirisque pour un système d'élevage totalement dépendant de la végétation spontanée ou cultivée (7). Au contraire, l'activité d'engraissement ne semble pas concurrencer le troupeau pour les ressources alimentaires naturelles puisqu'elle est effectuée à base de céréales secondaires achetées ou auto-produites. Les performances techniques de ces ateliers restent cependant modérées puisque Mahouachi et coll. (10) rapportent à Thala pour des agneaux âgés de six mois, pesant $23,4 \pm 4,1 \mathrm{~kg}$ au début de la phase d'engraissement, un gain moyen quotidien de $139 \mathrm{~g}$ par jour.

Cet élevage pseudo hors-sol présente plusieurs avantages pour les éleveurs qui le pratiquent. Il est peu contraignant, il utilise de la main d'œuvre familiale (enfants à l'école, femmes au foyer) et peut être très rentable si les cours de la viande sont propices. Ce qui détermine le plus ce type d'élevage c'est la disponibilité d'une assise financière qui permet d'acheter des animaux maigres sur le marché et d'atténuer le retard dans l'écoulement des produits. L'instauration d'une ligne de crédit pour soutenir cette spéculation pourrait avoir un impact très positif et améliorerait le niveau d'auto-approvisionnement en viandes rouges des filières ovines extensives.

Dans le contexte physique difficile à fortes contraintes économiques, la gestion de la composition des troupeaux n'obéit pas aux références techniques souvent prônées par les services techniques mais plutôt aux exigences de la conjoncture à très court terme. Le taux d'accroissement numérique des troupeaux de 7,05 p. 100 sur le site d'Ezzitouna a peut-être été occasionné, dans le cas de cette étude, par l'amélioration des conditions pluviométriques atteignant 396 et $401 \mathrm{~mm}$ pendant les campagnes agricoles respectivement de 1996 et 1997. Les faibles taux de réforme sont expliqués par l'attitude des éleveurs à ne pas décapitaliser, même lorsqu'il s'agit d'animaux improductifs. Pourtant, l'identification et l'élimination des animaux à faible productivité accroîtraient la productivité du troupeau. Elle réduirait la pression sur les ressources limitées préservant ainsi le statut nutritionnel des individus conservés.

\section{CONCLUSION}

L'augmentation des effectifs au cours des dernières décennies est l'élément déterminant de la stabilité du niveau de consommation des viandes rouges provenant des filières extensives (14). L'augmentation de la productivité, autrement dit du rendement indivi- 
duel des animaux utiles, est plus sujette à discussion car il existe peu d'indicateurs pour porter un jugement objectif. Afin de satisfaire les exigences quantitatives et qualitatives croissantes du consommateur dans un contexte où l'expansion horizontale pour ces filières n'est plus une option viable, les exemples d'intensification des élevages se multiplient. La question centrale pour les régions difficiles ciblées par cette étude est d'accroître les niveaux de productivité sans nuire aux fragiles écosystèmes semi-arides surtout pour ce qui concerne la préservation des sols et de la végétation naturelle. L'eau étant une ressource rare excluant l'irrigation à grande échelle, d'autres éléments devront être intégrés dans le système :

- la réhabilitation de l'élevage extensif sur parcours. Un appui plus important doit être consacré aux agriculteurs-éleveurs pour l'amélioration des parcours ou ce qu'il en reste. Ce qui est recherché n'est pas une augmentation des encouragements et des compensations mais plutôt une implication et une participation effective des bénéficiaires aux différentes phases de l'élaboration des programmes d'amélioration pastorale. Cette implication est importante dans le choix des espèces qui s'adaptent, dans l'emplacement des aménagements pastoraux par rapport au plan d'utilisation et aux aptitudes des parcelles, et dans les techniques d'exploitation des ressources pastorales par les animaux ;

- le développement de la capacité des services de vulgarisation dans les domaines de la production et de l'utilisation des ressources alimentaires pour le cheptel. La culture de l'orge dans l'interbande, le traitement à l'urée des pailles ou la confection des blocs alimentaires sont quelques exemples de technologies adaptées à la petite et moyenne exploitation. C'est le transfert de ces technologies qui ne semble pas être efficace ;

- la réorientation des programmes de développement de l'élevage et de sélection pour s'adresser à la petite et moyenne exploitation qui représente l'unité essentielle de la production. Très souvent, la mise en œuvre d'outils simples pour le pilotage de la gestion des ressources génétiques animales, au-delà de son impact immédiat sur la productivité du troupeau, peut engendrer des profits à long terme sur la production primaire (8);

- la mise en place de systèmes de crédit appropriés aux caractéristiques des exploitations de la zone de l'étude et facilement accessibles qui serviraient à diversifier les formes de production ovine comme, par exemple, les ateliers d'élevage et d'engraissement.

Ces recommandations méritent d'être reprises et réajustées si des données plus larges sont disponibles, prenant en compte les aspects liés aux contextes foncier, économique et social.

\section{Remerciements}

Des parties de ce travail ont été réalisées grâce au soutien de l'Institut de recherche pour le développement (Ird, ex-Orstom) en Tunisie et du projet « Highlands » de l'International Centre for Agricultural Research in Dry Areas (Icarda). Nous les remercions chaleureusement.

\section{BIBLIO GRAPH IE}

1. BAGNOULS F., GAUSSEN H., 1957. Climats biologiques et leur classification. Ann. Géogr., 355-LXVI : 193-220.

2. COOPER P.J.M., GREGORY P.J., TULLY D., HARRIS H.C., 1987. Improving water use efficiency of annual crops in the rainfed farming systems of W est Asia and N orth Africa. Expl. Agric., 23: 113-158.

3. DUPONT G., COMPERE R., 1997. Les systèmes d'élevage des moutons en Nouvelles-Galles du Sud (Australie). Biotechnol. Agron. Soc. Environ., $1: 34-38$.

4. EDDEBBARH A., HASSAR M., 1992. Caractérisation de l'élevage dans quelques systèmes céréaliers marocains. In: Proc. Joint ANPA, EAAP and ICAM AS Symposium, Livestock in the Mediterranean Cereal Production Systems, Rabat, M orocco, 7-10 O ctober 1990. (EAAP Publication N 0. 49)

5. EL AICH A., 1998. Impact of small ruminants on the environment in the southern shore of the Mediterranean. M éditerranée, 3 : 33-37.

6. Enquête nationale sur les structures des exploitations agricoles, 1995 Tunis, Tunisie, Direction générale de la planification et de développement des investissements agricoles (D gpdia), ministère de l'Agriculture.

7. FLAMANT J.C., 1992. Les systèmes d'élevage méditerranéens dans leurs rapports aux systèmes céréaliers : diversité et évolution. In: Proc Joint ANPA, EAAP and ICAMAS Symposium, Livestock in the Mediterranean Cereal Production Systems, Rabat, Morocco, 7-10 O ctober 1990. (EAAP Publication N o. 49)

8. GAY G.W., NOLTE D., BANNER R., BERGER Y., HAMOUDI M. 1990. Short-term gains in livestock management may lead to long-term range management benefits: a pilot programme in Morocco. London, UK, O verseas Development Institute. (Pastoral Development Network, paper 29c)
9. KAREM A., KSANTINI M., SCHO ENEN BERGER A., W AIBEL T., 1993. Contribution à la régénération de la végétation dans les parcs nationaux en Tunisie aride. Tunis, Tunisie, Deutsche Gtz.

10. MAHOUACHI M., REKIK M., ATTI N., CHERMITI A., M'HEDHBI K., 1999. Incorporation du tourteau de soja et/ou du tourteau de colza dans le concentré à base d'orge pour les agneaux des races $D$ ' $M$ an et à queue fine de l'O uest. Tropicultura (sous presse).

11. MAIDOUB A., MAHOUACHI M., YAHIAOUI A., RAHMANI D., 1994. Chemical composition and nutritive value of three barley cultivars grown under the semi-arid conditions of northwest Tunisia. Rachis, 13: 15-19.

12. NYGAARD D.F., AMIR P., 1987. Research strategies for development: Improving sheep and goat production in developing countries. In: Thomson E.F., Thomson F.S., Eds., Increasing small ruminant productivity in semi-arid areas. Dordrecht, the Netherlands, Kluwer Academic Publishers.

13. STEINFELD H., MAKI-HOKONNEN J., 1995. A classification of livestock production systems. World Anim. Rev., 84/85: 83-94.

14. Stratégie pour le développement des parcours en zones arides et semi-arides, Annexe III. Rapport technique : Tunisie, 1995. Washington, DC, Etats-U nis, Banque mondiale, Departement Maghreb et Iran, bureau régional M oyen-O rient et Afrique du N ord.

15. VAILLANT G., 1979. L'élevage en Tunisie, bilan à la fin du IVe plan et perspectives. Thèse D oct. M éd. vét., Env, M aisons-Alfort, France.

Accepté le 29.06.2001 


\section{Summary}

Rekik M., Mahouachi M., Gharbi M., Attia W., Medhioub L. The Dilemma of Extensive Sheep Production Systems in the $\mathrm{H}$ ighlands of the Semiarid N orthwest of Tunisia

To characterize extensive sheep production systems in the semiarid bioclimate of Northwest Tunisia, two mountain sites were selected. The production systems were sedentary and in close relationship with cereal production. Barley, along with the practice of fallowing on low-fertility sloping lands, had replaced rangelands. The flocks were usually small and increasingly confined to smaller spaces. They were dependent on poorly efficient feed strategies and characterized by low technical-economical performances. In addition, farmers' financial support of their flocks was precarious and the farmers themselves depended on climatic and economical changes. Faced with the space extension and small flock size problems, it seems necessary to increase sheep productivity in these areas. Recommendations that take into account the particular geographical, technical and economical context of the farms are proposed.

Key words: Sheep - Cereal - Extensive husbandry - Animal production - Semiarid climate - Tunisia.

\section{Resumen}

Rekik M., Mahouachi M., Gharbi M., Attia W., Medhioub L. Dilema de la crianza ovina extensiva en las regiones altas del nordeste, semi árido tunecino

Se escogieron dos sitios de las regiones montañosas, con bioclima semi árido en el nordeste de Túnez, con el fin de caracterizar los sistemas extensivos de la producción ovina. Los sistemas de producción encontrados son de tipo sedentario, estrechamente unidos a la producción de cereales y por los cuales se sacrifico el uso de trayectos, en beneficio de una extensión del cultivo de la cebada, acompañada de una utilización de los añojales en pendientes de baja fertilidad. Los hatos, generalmente pequeños y confinados cada vez más a espacios más reducidos, recurren a estrategias alimenticias poco eficaces, con rendimientos técnico económicos bajos. Esta producción se encuentra también minusválida por la frágil base financiera de los criadores-agricultores y por la vulnerabilidad frente al contexto climático y económico. Frente a las dificultades en la extensión del espacio o del crecimiento de los efectivos, la cría ovina en estas zonas se enfrenta a la necesidad de aumentar la productividad. Para esto, se proponen recomendaciones que toman en cuenta el contexto técnico económico particular de los establecimientos agrícolas de la zona.

Palabras clave: Ovino - Cereal - Crianza extensiva - Producción animal - Clima semiárido - Túnez. 